\title{
EVOLUTIONARY COMPUTATION THEORY AND APPLICATIONS
}




\section{This page is intentionally left blank}




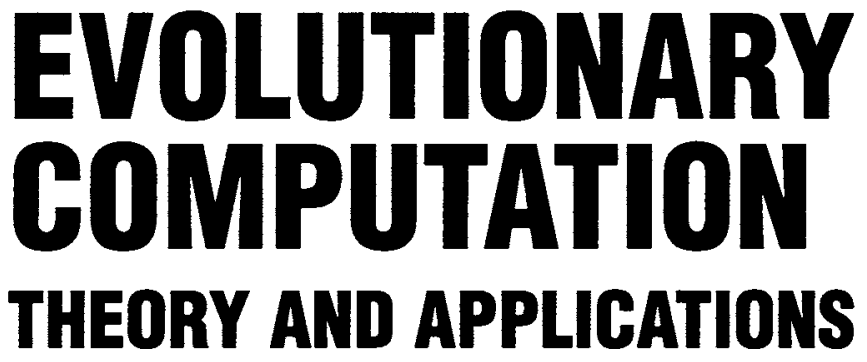

Editor

Xin Yao

University of Birmingham, UK 


\section{Published by}

World Scientific Publishing Co. Pte. Ltd.

P O Box 128, Farrer Road, Singapore 912805

USA office: Suite 1B, 1060 Main Street, River Edge, NJ 07661

UK office: 57 Shelton Street, Covent Garden, London WC2H 9HE

\section{British Library Cataloguing-in-Publication Data \\ A catalogue record for this book is available from the British Library.}

\section{EVOLUTIONARY COMPUTATION: THEORY AND APPLICATIONS}

Copyright $\odot 1999$ by World Scientific Publishing Co. Pte. Ltd.

All rights reserved. This book, or parts thereof, may not be reproduced in any form or by any means, electronic or mechanical, including photocopying, recording or any information storage and retrieval system now known or to be invented, without written permission from the Publisher.

For photocopying of material in this volume, please pay a copying fee through the Copyright Clearance Center, Inc., 222 Rosewood Drive, Danvers, MA 01923, USA. In this case permission to photocopy is not required from the publisher.

ISBN 981-02-2306-4

Printed in Singapore. 


\section{Preface}

Evolutionary computation is the study of computational systems which use ideas and get inspiration from natural evolution and adaptation. It includes a wide range of topics. This book covers some of the most interesting research areas in evolutionary computation. It deals with both theories and applications. It is a self-contained volume which covers both introductory material and selected advanced topics.

Chapter 1 introduces the field of evolutionary computation. It reviews briefly the history and different branches of evolutionary computation. It also gives an overview of various applications of evolutionary computation techniques. Colombetti and Dorigo report their work on using ALECSYS to develop behavioral modules for mobile robots and discuss the potential advantages of using the evolutionary approach in behavioral engineering in Chapter 2. Schmidhuber describes a theoretical foundation for multi-agent learning and incremental selfimprovement in an unrestricted environment in Chapter 3. Wah and Ieumwananonthachai introduce TEACHER, a genetics-based system for learning and generalising heuristics under resource constraints, in Chapter 4. Koza and Andre describe the automatic discovery of protein motifs using genetic programming in Chapter 5. Tsoi and Shaw present many results on emerging complex behaviors from multiple insect simulation in Chapter 6. Fukuda et al. introduce a new virus-evolutionary genetic algorithm and present the experimental results of its application to the traveling salesman problem in Chapter 7. Kim and Myung describe a hybrid evolutionary optimisation algorithm for constrained numerical optimisation problems in Chapter 8. de Garis discusses his dream machine, i.e., CAM-BRAIN, in Chapter 9. Finally, Yao and Darwen present some experiments on evolving strategies for $N$-person 
iterated prisoner's dilemma games (where $2 \leq N \leq 8$ ) in Chapter 10 .

This book does not require previous background or experience in the field of evolutionary computation since introductory material is included in the book. It may be used as a textbook at the graduate or senior undergraduate level, or as a reference book for people interested in evolutionary computation. 


\section{Acknowledgements}

This book was originally planned for publication in late 1995 . However, it was delayed several times due to personal reasons. I would like to apologise to anyone, especially the authors, who has been affected by this delay. I am grateful to the editors from the World Scientific Publ. Co. and authors of individual chapters for their patience and understanding while I was putting the book together. 


\section{This page is intentionally left blank}




\section{List of Contributors}

Marco Colombetti

Artificial Intelligence and Robotics Project

Dipartimento di Elettronica e Informazione

Politecnico di Milano

Piazza Leonardo da Vinci, 32

20133 Milano, Italy

Email: colombet@elet.polimi.it

URL: http://www.elet.polimi.it/people/colombet/

Marco Dorigo

Chercheur Qualifie' du FNRS

IRIDIA CP 194/6

Universite' Libre de Bruxelles

Avenue Franklin Roosevelt 50

1050 Bruxelles, Belgium

Email: mdorigo@ulb.ac.be

URL: http://iridia.ulb.ac.be/dorigo/dorigo.html

Jürgen Schmidhuber

IDSIA, Corso Elvezia 36

CH-6900-Lugano, Switzerland

Email: juergen@idsia.ch

URL: http://www.idsia.ch/ juergen 
Benjamin W. Wah

Department of Electrical and Computer Engineering and the Coordinated Science Laboratory

University of Illinois at Urbana-Champaign 1308 West Main Street, Urbana, IL61801, USA

Email: wah@manip.crhc.uiuc.edu

URL: http://manip.crhc.uiuc.edu

Arthur Ieumwananonthachai

Department of Electrical and Computer Engineering and the Coordinated Science Laboratory

University of Illinois at Rubana-Champaign

1308 West Main Street, Urbana, IL61801, USA

Email: arthuri@manip.crhc.uiuc.edu

URL: http://manip.crhc.uiuc.edu

John Koza

Department of Computer Science

Standford University

Stanford, CA 94305, USA

Email: koza@cs.stanford.edu

URL: http://www-cs-faculty.cs.stanford.edu/ koza

David Andre

Computer Science Division

Room 387, Soda Hall, \#1776

University of California at Berkeley

Berkeley, CA 94720-1776, USA

Email: dandre@cs.berkeley.edu

URL: http://http.cs.berkeley.edu/ dandre

Ah Chung Tsoi

Faculty of Informatics

University of Wollongong

NSW, Australia

Email: act@uow.edu.au

URL: http://www.uow.edu.au/informatics/facultystaff.html 
Jeff Shaw

Department of Electrical and Computer Engineering

University of Queensland

St Lucia, Queensland 4072, Australia

Toshio Fukuda

Department of Micro System Engineering

Nagoya University

Furo-cho, Chikusa-ku, Nagoya, 464-01, Japan

Email: fukuda@mein.nagoya-u.ac.jp

URL: http://www.mein.nagoya-u.ac.jp/staff/fukuda-e.html

N. Kubota

Department of Micro System Engineering

Nagoya University

Furo-cho, Chikusa-ku, Nagoya, 464-01, Japan

K. Shimojima

Department of Micro System Engineering

Nagoya University

Furo-cho, Chikusa-ku, Nagoya, 464-01, Japan

Jong-Hwan Kim

Department of Electrical Engineering

Korea Advanced Institute of Science and Technology

373-1, Kusung-dong, Yusung-gu, Taejon-shi

305-701, Republic of Korea

Email: johkim@vivaldi.kaist.ac.kr

URL: http://www-ee.kaist.ac.kr/department/professors/jhkim.html

H. Myung

Department of Electrical Engineering

Korea Advanced Institute of Science and Technology

373-1, Kusung-dong, Yusung-gu, Taejon-shi

305-701, Republic of Korea

Email: myung@vivaldi.kaist.ac.kr 
Hugo de Garis

Evolutionary Systems Department

ATR Human Information Processing Laboratories

2-2 Kikari-dai, Seika-cho, Soraku-gun

Kansai Science City, Kyoto-fu, 619-02, Japan

Email: degaris@hip.atr.co.jp

URL: http://www.hip.atr.co.jp/ degaris

\section{Paul Darwen}

Department of Computer Science and Electrical Engineering The University of Quensland

Brisbane, QLD 4072. Australia

Email: darwen@csee.uq.edu.au

URL: http://www.csee.uq.edu.au/ darwen 


\section{Contents}

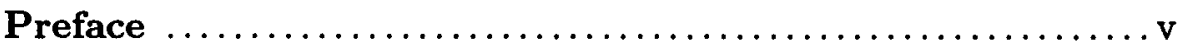

Acknowledgements $\ldots \ldots \ldots \ldots \ldots \ldots \ldots \ldots \ldots \ldots \ldots \ldots \ldots$ vii

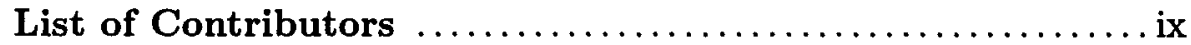

1 Introduction

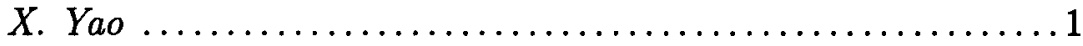

2 Evolutionary Computation in Behavior Engineering

M. Colombetti and M. Dorigo ........................ 37

3 A General Method for Incremental Self-improvement and Multi-agent Learning

J. Schmidhuber ...................................81

4 Teacher: A Genetics-Based System for Learning and for Generalizing Heuristics

B. W. Wah and A. Ieumwananonthachai .................124 
5 Automatic Discovery of Protein Motifs Using Genetic Programming

J. R. Koza and D. Andre ........................171

6 The Role of Self Organization in Evolutionary Computations A. C. Tsoi and J. Shaw ............................ 198

7 Virus-Evolutionary Genetic Algorithm and Its Application to Traveling Salesman Problem

T. Fukuda, N. Kubota, and K. Shimojima ..............235

8 Hybrid Evolutionary Optimization Algorithm for Constrained Problems

J.-H. Kim and H. Myung ....................... 256

9 CAM-BRAIN - The Evolutionary Engineering of a Billion Neuron Artificial Brain H. de Garis .................................296

10 An Evolutionary Approach to the N-Player Iterated Prisoner's Dilemma Game $X$. Yao and P. Darwen ............................... 331

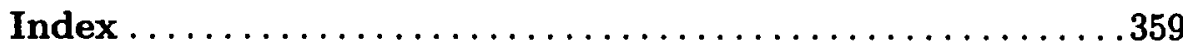

